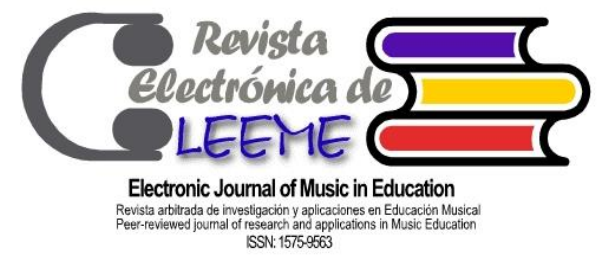

\title{
Educación musical y Aprendizaje-Servicio: estudio de caso en la formación de los futuros docentes de Educación Primaria
}

\author{
Music education and service-learning: a case study in Primary Education teacher \\ training
}

\author{
Carol Gillanders \\ carol.gillanders@usc.es \\ Departamento de Didácticas Aplicadas \\ Universidad de Santiago de Compostela \\ Santiago de Compostela, España \\ ORCID: http://orcid.org/0000-0001-7122-5416 \\ Antía Cores Torres \\ antia.cores.torres@usc.es \\ Departamento de Pedagogía y Didáctica \\ Universidad de Santiago de Compostela \\ Santiago de Compostela, España \\ ORCID: http://orcid.org/0000-0002-4152-9889
}

\author{
Laura Tojeiro Pérez \\ laura.tojeiro@usc.es \\ Departamento de Didácticas Aplicadas \\ Universidad de Santiago de Compostela \\ Santiago de Compostela, España \\ ORCID: http://orcid.org/0000-0003-4229-3844
}

doi: 10.7203/LEEME.42.12329

Recibido: 28-03-2016 Aceptado: 11-10-2018. Contacto y correspondencia: Carol Gillanders, Departamento de Didácticas Aplicadas Universidad de Santiago de Compostela, Avda. Xoán XXI, s/n. Campus Norte, 15782 Santiago de Compostela. España.

Resumen

En este artículo, se presenta un estudio de caso que pretende analizar el impacto de la implantación de un proyecto de AprendizajeServicio en la materia de 'Música en la Educación Primaria' en la formación de los futuros docentes de Educación Primaria en la Universidad de Santiago de Compostela. Este proyecto abarcó el diseño, impartición y evaluación de talleres de iniciación musical en locales socioculturales del contexto rural para niños y niñas de edades comprendidas entre los seis y los doce años. Los futuros graduados pudieron desarrollar competencias propias del título que estaban cursando, así como conocer otros ámbitos de desarrollo profesional diferentes de la educación reglada.

Palabras clave: educación musical, Aprendizaje-Servicio, formación del profesorado, locales socioculturales del rural.

Abstract

The case study presented in this article aims to analyse the impact of the implementation of a service-learning project in the subject 'Music for Primary Education' has in primary teacher training at the University of Santiago de Compostela. This project covered the design, teaching, and assessment of music workshops in rural sociocultural premises for girls and boys between six and ten years old. The future teachers have been able to develop competencies appropriate for their degree programs, and they have been able to familiarize themselves with fields of professional development that differ from those offered in their formal programs.

Key words: music education, service-learning, teacher training, rural sociocultural premises. 


\section{Introducción}

El Informe sobre el estado de la Cultura en España 2016. La cultura como motor de cambio encargado por la Fundación Alternativas hace hincapié en los crecientes desequilibrios en el acceso a la cultura de la población española. Sin lugar a dudas, en el siglo XXI ya no es cuestionable la importancia del contacto con la música a edades tempranas para la formación integral del individuo: diferentes informes e investigaciones así lo constatan (Bresler, 2002; Ewing, 2010; Mark, 2002; Rauscher, 2009). Evidencias de los efectos positivos de la participación en actividades artísticas son proporcionadas, por ejemplo, en los trabajos de Fiske (1999) y Deasy (2002). Además de su influencia en aspectos cognitivos y académicos, la educación musical también tiene un impacto beneficioso en el ámbito personal (bienestar, aspectos emocionales e identitarios) o social (mejora de la convivencia, responsabilidad social) (Carrillo, Viladot y PérezMoreno, 2017).

Con el fin de garantizar un acceso equitativo a la cultura en los distintos núcleos de población, de brindar un contacto directo con la música y de facilitar que los niños y niñas de los distintos ámbitos culturales se puedan enriquecer de sus beneficios, consideramos que la inclusión del Aprendizaje-Servicio (en adelante, ApS) en la formación de los futuros docentes puede constituir una herramienta ideal para conseguir estos logros.

En los últimos años se ha producido en España un creciente interés por el Aprendizajeservicio en el ámbito de la enseñanza superior. Aunque realizar una definición de ApS presenta sus dificultades dado sus diferentes enfoques (Sigmon, 1997; Ugarte y Naval, 2010), podemos definirlo como una metodología "que combina procesos de aprendizaje y servicio a la comunidad en un solo proyecto bien articulado en el que los participantes aprenden a trabajar sobre las necesidades reales del entorno con la finalidad de mejorarlo" (Puig Rovira, Batlle, Bosch y Palos, 2006, p. 22). Si bien oficialmente el término "service-learning" se utiliza solamente desde el año 1967 (Santos Rego, Sotelino Losada y Lorenzo Moledo, 2015), puede decirse que tiene una larga tradición en algunos contextos como el anglosajón (Kezar y Rhoads, 2001). En nuestra universidad, ha comenzado recientemente su proceso de institucionalización a través de la oferta de cursos para el profesorado y del respaldo a las iniciativas emprendidas por los docentes, facilitando su tarea por medio del Servicio de Participación e Integración Universitaria (SEPIU) y el apoyo del grupo de investigación ESCULCA. El SEPIU colabora con el profesorado en la búsqueda de socios y/o firma de convenios de partenariado o en la tramitación del seguro de accidentes para los estudiantes, entre otros.

El ApS estimula a los estudiantes incrementando su interés por la materia y su sentido de la responsabilidad cívica, proporcionándoles un espacio para su participación activa en la sociedad y permitiendo un aprendizaje colaborativo, un desarrollo personal y profesional, a la vez que torna más placentera la docencia para el profesorado (Bringle y Hatcher, 1996; 2000). Como señalan Puig Rovira, Gijón Casares, Martín García y Rubio Serrano (2011) mediante el ApS se promueve el desarrollo de competencias reflexivas y críticas, el compromiso y la responsabilidad. Apuntan Lorenzo, Mella, García y Varela (2017) que

CAROL GILLANDERS, ANTIA CORES TORRES Y LAURA TOJEIRO PÉREZ. THE CONTENT OF THIS ARTICLE IS THE SOLE RESPONSIBILITY OF THE AUTHORS. THE REVISTA ELECTRÓNICA DE LEEME AND UNIVERSITAT DE VALËNCIA ARE NOT LIABLE FOR ANY LEGAL ACTIONS THAT MAY ARISE INVOLVING THE ARTICLE'S CONTENT. REVISTA ELECTRÓNICA DE LEEME - UISTA ELECTRÓNICA EUROPEA DE MÚSICA EN LA EDUCACIÓN-HTTP://OJS.UV.ES/INDEX.PHP/LEEME/INDEX ISSN: 1575-9563. EDITORES: UNIVERSIDAD DE VALENCIA Y JESÚS TEJADA GIMÉNEZ. VISIBILIDAD DE ESTA REVISTA: SCOPUS, EMERGING CITATION INDEX (CLARIVATE), EBSCO, CINDOC (CSIC), CITEFACTOR, COPAC, DIALNET, DICE (CSIC), DOAJ, E-REVISTAS (CSIC), EBSCO PREMIER, ERIH+, GALE CENGAGE LEARNING, IN-RECS, IRESIE, LATINDEX, MIAR, OCLC WORLDCAT, RESH, REDIB, RILM CORE JOURNALS, SUDOC, ULRICHS, ESTA REVISTA
ESTÁ PUBLICADA CON EL APOYO INSTITUCIONAL DE REDIRIS-CONSEJO SUPERIOR DE INVESTIGACIONES CIENTIFICAS Y ES DE ACCESO LIBRE. CREATIVE COMMONS LICENSE 4.0 BY 
"El efecto y los beneficios del aprendizaje servicio pueden ir más allá de los estudiantes y de los potenciales resultados cognitivo-sociales en los mismos, ya que es posible que alcancen al profesorado, a la comunidad y a la universidad en su conjunto, máxime si consigue vincular tres de sus inequívocas misiones: docencia, investigación y responsabilidad social” (p. 122).

En el ámbito específico de la educación musical, Siebenaler (2005) señala la importancia de llevar a cabo proyectos de ApS con alumnado generalista, para que a través de la reflexión personal y la propia práctica como docentes puedan detectar sus debilidades y fortalezas y "aplicar las estrategias, técnicas, y habilidades presentadas y practicadas en clase" (p. 6). En su caso, el alumnado pudo trabajar diferentes áreas del currículum a través de la música. También en la Península Ibérica existen trabajos en esta línea: por ejemplo, la colaboración con diferentes asociaciones y colectivos que posibilitó una serie de performances que desencadenaban reflexiones sobre algunas problemáticas sociales con alumnado universitario de la asignatura 'Didáctica de la Música' (Riaño Galán, Mier Pérez y Pozo Miranda, 2017); el proyecto llevado a cabo en el CEIP El Castell de Albalat dels Sorells (Valencia) con alumnado de Pimaria que participó en un coro escolar (Sáez Santa Eulalia y Marín Suelves, 2016); o la intervención sociocultural en la comunidad de Contige (Portugal) a través de la creación de la Tuna ContiTuna y la recuperación del patrimonio popular musical (Sadio-Ramos y Ortiz-Molina, 2018). En la propia página de la Red Española de Aprendizaje-Servicio, podemos encontrar algunos proyectos relacionados con la música u otras temáticas que nos sirven de inspiración (https://aprendizajeservicio.net/finalistas-premios-aps-2017/), como por ejemplo Cantem Junts (proyecto musical intergeneracional) o Contacontes: La rateta que llegia a l'escaleta (escenificación de un cuento tradicional fuera del contexto escolar habitual), entre otros.

En su análisis de documentos sobre la educación musical emitidos por la Unión Europea, López García y De Moya Martínez (2017) indican que no existe concordancia entre las proclamaciones a nivel europeo que defienden la inclusión de actividades artísticas en los sistemas educativos y las legislaciones nacionales al respecto. Lamentablemente, a pesar de lo que demuestran las investigaciones sobre los beneficios de las experiencias musicales (Giordanelli, 2011), la asignación horaria dedicada a la música en la Educación Primaria sigue siendo muy escasa (en Galicia, una hora semanal) o inexistente, al establecerse esta posibilidad a partir de la LOMCE. Lo mismo podemos decir de la presencia de la música en los planes de estudio del futuro profesorado de Educación Primaria, así lo advierten López García, Madrid Vivar y De Moya Martínez (2017) al afirmar que

"El perfil del Maestro Generalista ha vuelto a ocupar un papel fundamental, mientras que las antiguas especialidades han perdido peso específico pasando a denominarse menciones cualificadoras. Esto ha supuesto una drástica reducción de créditos ECTS relacionados con la formación musical" (p. 437).

Rosa Napal (2015) constata en su estudio sobre la formación musical de los futuros docentes de Educación Primaria de Galicia, la insuficiente preparación que presenta el alumnado en relación a la educación musical: "es una realidad que evidencia la existencia de problemas de índole general en cuanto a la educación musical en las etapas educativas pre-universitarias" (p. 312). También, así lo señala Bautista Cupul (2017) en su investigación sobre la formación musical

CAROL GILLANDERS, ANTIA CORES TORRES Y LAURA TOJEIRO PÉREZ. THE CONTENT OF THIS ARTICLE IS THE SOLE RESPONSIBILITY OF THE AUTHORS. THE REVISTA ELECTRÓNICA DE LEEME AND UNIVERSITAT DE VALËNCIA ARE NOT LIABLE FOR ANY LEGAL ACTIONS THAT MAY ARISE INVOLVING THE ARTICLE'S CONTENT. REVISTA ELECTRÓNICA DE LEEME - ISTA ELECTRÓNICA EUROPEA DE MÚSICA EN LA EDUCACIÓN-HTTP://OJS.UV.ES/INDEX.PHP/LEEME/INDEX ISSN: 1575-9563. EDITORES: UNIVERSIDAD DE VALENCIA Y JESÚS TEJADA GIMÉNEZ. VISIBILIDAD DE ESTA REVISTA: SCOPUS, EMERGING CITATION INDEX (CLARIVATE), EBSCO, CINDOC (CSIC), CITEFACTOR, COPAC, DIALNET, DICE (CSIC), DOAJ, E-REVISTAS (CSIC), EBSCO PREMIER, ERIH+, GALE CENGAGE LEARNING, IN-RECS, IRESIE, LATINDEX, MIAR, OCLC WORLDCAT, RESH, REDIB, RILM CORE JOURNALS, SUDOC, ULRICHS, ESTA REVISTA
ESTÁ PUBLICADA CON EL APOYO INSTITUCIONAL DE REDIRIS-CONSEJO SUPERIOR DE INVESTIGACIONES CIENTIFICAS Y ES DE ACCESO LIBRE. CREATIVE COMMONS LICENSE 4. B BY 
adquirida por los estudiantes al afirmar que éstos "valoran no sentirse preparados para utilizar la música en el aula" (p. 32) siendo necesario establecer un debate sobre la orientación y los contenidos fundamentales a impartir en la asignatura de Música. Hennessy (2000) sugiere que es necesario ofrecer a los estudiantes oportunidades para enseñar y realizar devoluciones constructivas y positivas a las tareas que desempeñen, ya que sus actitudes y experiencias previas relacionadas con la música influyen en su seguridad para incluirla en su práctica docente. Compartimos la opinión de Burke (2016), que recomienda realizar una labor de acompañamiento a los educadores sin una experiencia artística (en su caso, crea a tal fin una web) y de proporcionarles una formación adecuada, porque la calidad de la experiencia artística vivida influye en la calidad de la educación artística que se imparte (Bamford, 2009). Dogani (2008) propone promover la reflexión en el aula, puesto que a través de ésta los futuros docentes generalistas se pueden enfrentar a aquellos aspectos que les causan inseguridad al enseñar música.

En vista de lo expuesto anteriormente, pensamos que la participación en talleres de iniciación musical puede potenciar el desarrollo de competencias y habilidades necesarias en todo docente de Educación Primaria, propiciar que el alumnado de la materia 'Música en la Educación Primaria' conozca otros ámbitos de desarrollo profesional (educación sociocomunitaria y sociocultural), favorecer la percepción de los estudiantes sobre la materia, y ofrecer un programa de calidad basado en actividades de iniciación musical para un entorno que manifiesta la necesidad de dicho servicio.

Este proyecto tiene como principal objetivo que el alumnado del Grado de maestro/a en Educación Primaria desarrolle algunas de las competencias y habilidades necesarias para ser docente reflejadas en la Memoria del título tales como: el diseño, la planificación y la impartición de diferentes sesiones de educación musical, la gestión y control de un grupo de alumnado con edades e intereses distintos, la toma de decisiones en contextos cambiantes y de incertidumbre, la adecuación de los contenidos a las demandas de los participantes y la evaluación de su propia práctica.

En este artículo, se presenta un estudio de caso basado en el proyecto de ApS "Talleres de iniciación musical", que fue desarrollado durante los cursos académicos 2015-16, 2016-17 y 2017-18 en la materia de 'Música en la Educación Primaria' del Grado de Maestro/a en Educación Primaria de la Universidad de Santiago de Compostela. Estos talleres tuvieron lugar en el medio rural. El Ayuntamiento de Santiago de Compostela cuenta con cuarenta y tres centros socioculturales en diferentes zonas, de los cuales veinte están ubicados en zonas rurales. En estos centros, se realizan diferentes conferencias, charlas, conciertos y actividades para los usuarios de todas las edades, pero no en todos se ofertan talleres artísticos. Se partió, por tanto, "de un análisis de las necesidades sociales del entorno inmediato, en donde se planificó un plan de servicio con el objetivo final de mejorar la situación. "El servicio será real y se hará con el mayor rigor posible" (Santos Rego et al., 2015, p. 20) y las actividades que se propusieron para estos talleres incluyeron sonorizaciones, juegos de movimiento, percusión corporal, bailes, construcción de cotidiáfonos y exploración de instrumentos.

CAROL GILLANDERS, ANTIA CORES TORRES Y LAURA TOJEIRO PÉREZ. THE CONTENT OF THIS ARTICLE IS THE SOLE RESPONSIBILITY OF THE AUTHORS. THE REVISTA ELECTRÓNICA DE LEEME AND UNIVERSITAT DE VALËNCIA ARE NOT LIABLE FOR ANY LEGAL ACTIONS THAT MAY ARISE INVOLVING THE ARTICLE'S CONTENT. REVISTA ELECTRÓNICA DE LEEME - ISTA ELECTRÓNICA EUROPEA DE MÚSICA EN LA EDUCACIÓN-HTTP://OJS.UV.ES/INDEX.PHP/LEEME/INDEX ISSN: 1575-9563. EDITORES: UNIVERSIDAD DE VALENCIA Y JESÚS TEJADA GIMÉNEZ. VISIBILIDAD DE ESTA REVISTA: SCOPUS, EMERGING CITATION INDEX (CLARIVATE), EBSCO, CINDOC (CSIC), CITEFACTOR, COPAC, DIALNET, DICE (CSIC), DOAJ, E-REVISTAS (CSIC), EBSCO PREMIER, ERIH+, GALE CENGAGE LEARNING, IN-RECS, IRESIE, LATINDEX, MIAR, OCLC WORLDCAT, RESH, REDIB, RILM CORE JOURNALS, SUDOC, ULRICHS, ESTA REVISTA
ESTÁ PUBLICADA CON EL APOYO INSTITUCIONAL DE REDIRIS-CONSEJO SUPERIOR DE INVESTIGACIONES CIENTIFICAS Y ES DE ACCESO LIBRE. CREATIVE COMMONS LICENSE 4.0 BY 


\section{Método}

El proyecto 'Talleres de iniciación musical' pretendió incorporar a la materia obligatoria 'Música en la Educación Primaria' del Grado de maestro/a en Educación Primaria actividades que acercasen al alumnado a contextos reales de práctica docente, a través de un proyecto de ApS desarrollado en diferentes locales socioculturales en contextos rurales de Santiago de Compostela. Mediante un estudio de caso se analiza el impacto de este programa en la formación de los estudiantes universitarios. Éste es definido por Creswell (1998) como "la exploración de un "sistema cerrado" o de un caso (o múltiples casos) a través del tiempo mediante la recolección detallada de información que involucra múltiples fuentes de información ricas en contexto" (p. $61)$.

Durante el estudio se recogió información sobre las actuaciones y percepciones del alumnado a través de la observación participante, video-grabaciones, fotografías, ruedas de opinión y cuestionarios de evaluación a fin de analizar el programa implementado. Posteriormente, se procedió a triangular todos los datos obtenidos a través del análisis de los documentos audiovisuales tratando de determinar fortalezas y debilidades y extrayendo códigos recurrentes de las producciones escritas (cuestionarios) e intervenciones orales (ruedas de opinión) de los participantes, tanto de niños y niñas como de estudiantes de grado.

Para los estudiantes universitarios, el objetivo de la participación en este programa fue desarrollar las competencias incluidas en la memoria de la titulación:

G10 - Reflexionar sobre las prácticas de aula para innovar y mejorar la labor docente. Adquirir hábitos y destrezas para el aprendizaje autónomo y cooperativo y promoverlo entre los estudiantes.

E54 - Comprender los principios que contribuyen a la formación cultural, personal y social desde las artes.

E55 - Conocer el currículo escolar de la educación artística, en sus aspectos plástico, audiovisual y musical.

E57 - Desarrollar y evaluar contenidos del currículo mediante recursos didácticos apropiados y promover las competencias correspondientes en los estudiantes (Memoria del título, 2011, p. 6-8).

\subsection{Los participantes}

En este estudio, participaron un total de treinta estudiantes del Grado de Maestro/a en Educación Infantil y Primaria, veintitrés niños y niñas y tres docentes (Tabla 1). El 'Taller de iniciación musical' se ofertó por primera vez durante el curso 2015/16 a un grupo de niños y niñas de seis a doce años de edad ( $1^{\circ}-6^{\circ}$ de Educación Primaria) en el Local Sociocultural del Rural (en adelante, LSC) Villestro. Las encargadas de impartir las siete sesiones (una hora semanal durante los meses de abril y mayo de 2016) fueron tres alumnas de la materia 'Música en la Educación Primaria’ del tercer curso del Grado de Maestro/a de Educación Primaria, junto con la

CAROL GILLANDERS, ANTIA CORES TORRES Y LAURA TOJEIRO PÉREZ. THE CONTENT OF THIS ARTICLE IS THE SOLE RESPONSIBILITY OF THE AUTHORS. THE REVISTA ELECTRÓNICA DE LEEME AND UNIVERSITAT DE VALËNCIA ARE NOT LIABLE FOR ANY LEGAL ACTIONS THAT MAY ARISE INVOLVING THE ARTICLE'S CONTENT. REVISTA ELECTRÓNICA DE LEEME - ISTA ELECTRÓNICA EUROPEA DE MÚSICA EN LA EDUCACIÓN-HTTP://OJS.UV.ES/INDEX.PHP/LEEME/INDEX ISSN: 1575-9563. EDITORES: UNIVERSIDAD DE VALENCIA Y JESÚS TEJADA GIMÉNEZ. VISIBILIDAD DE ESTA REVISTA: SCOPUS, EMERGING CITATION INDEX (CLARIVATE), EBSCO, CINDOC (CSIC), CITEFACTOR, COPAC, DIALNET, DICE (CSIC), DOAJ, E-REVISTAS (CSIC), EBSCO PREMIER, ERIH+, GALE CENGAGE LEARNING, IN-RECS, IRESIE, LATINDEX, MIAR, OCLC WORLDCAT, RESH, REDIB, RILM CORE JOURNALS, SUDOC, ULRICHS, ESTA REVISTA
ESTÁ PUBLICADA CON EL APOYO INSTITUCIONAL DE REDIRIS-CONSEJO SUPERIOR DE INVESTIGACIONES CIENTIFICAS Y ES DE ACCESO LIBRE. CREATIVE COMMONS LICENSE 4.0 BY 
coordinadora del taller, alumna de cuarto curso de la mención en Educación Musical, todas con estudios profesionales de música.

En el curso siguiente, el taller tuvo lugar en el LSC Figueiras y en él participaron cuatro niñas y dos niños de entre 8 y 10 años $\left(3^{\circ}\right.$ y $4^{\circ}$ de Educación Primaria). El taller fue impartido durante los meses de abril y mayo de 2017 por ocho alumnas y un alumno de la materia de tercer curso "Música en la Educación Primaria". A diferencia de la edición anterior, en este curso el alumnado no tenía estudios reglados de música, aunque sí es cierto que poseían formación en baile tradicional gallego y un participante era monitor de tiempo libre.

Por último, el tercer taller llevado a cabo en el LSC A Peregrina durante los meses de octubre, noviembre y diciembre de 2017 contó con la participación de trece alumnas de la materia 'Música en la Educación Primaria', cuatro de las cuales tenían experiencia en baile tradicional gallego y dos poseían estudios reglados de música; y cuatro alumnas de la materia 'Motricidad Infantil' del Grado de Maestro/a en Educación Infantil, también con experiencia en baile gallego. En esta edición, asistieron regularmente ocho niños y niñas de la zona.

Tabla 1. Participantes en el proyecto 'Taller de iniciación musical'

\begin{tabular}{llccc}
\hline & & \multicolumn{3}{c}{ PARTICIPANTES } \\
\cline { 3 - 5 } & & NIÑOS/AS & ALUMNADO & PROFESORADO \\
& & & UNIVERSITARIO & UNIVERSITARIO \\
\hline VILLESTRO & ABRIL-MAYO 2016 & 6 & 4 & 1 \\
FIGUEIRAS & ABRIL-MAYO 2017 & 6 & 9 & 2 \\
A PEREGRINA & OCTUBRE-DIC. 2017 & 8 & 17 & 3 \\
\hline
\end{tabular}

Fuente: Elaboración propia

\subsection{Los proyectos}

El desarrollo de los tres proyectos fue posible gracias a la colaboración de la responsable de los Locales Socioculturales del Rural de la ciudad de Santiago de Compostela, quien se encargó de buscar los espacios para desarrollar las actividades, imprimir los carteles de difusión, realizar la publicidad en las redes sociales, matricular a las personas interesadas y solicitar la autorización de padres/madres/tutores legales para la grabación de vídeos y fotografías.

Como señala Puig Rovira et al. (2011), los proyectos de ApS son el resultado de un proceso que pasa por diferentes fases. En todos nuestros proyectos, podemos distinguir tres: diseño y coordinación de los talleres, implementación y evaluación de los mismos.

\subsubsection{Primera fase: diseño y coordinación de los talleres}

En esta fase, se presentó el proyecto al alumnado de la materia y se abrió un período de quince días para que, de forma voluntaria, indicaran si estaban interesados o no y se apuntasen en una lista provisional, siendo todos seleccionados. También, se preguntó por su bagaje musical y

CAROL GILLANDERS, ANTIA CORES TORRES Y LAURA TOJEIRO PÉREZ. THE CONTENT OF THIS ARTICLE IS THE SOLE RESPONSIBILITY OF THE AUTHORS. THE REVISTA ELECTRÓNICA DE LEEME AND UNVERSITAT DE VALENCIA ARE NOT LARLE FOR ANYLEGAL ACTIONS THAT MAY ARISE INVOLVING THE ARTICLE'S CONTENT. REVISTA ELECTRÓNICA DEELEEME - ISTA ELECTRÓNICA EUROPEA DE MÚSICA EN LA EDUCACION-HTTP:/ / OSSUVES/INDEX PHP/LEEME/INDEXISSN" 1575-9563. EDITORES" UNIVERSIDAD DE VALENCIA Y JESÚS TEJADA GIMÉNEZ. VISIBILIDAD DE ESTA REVISTA: SCOPUS, EMERGING CITATION INDEX (CLARIVATE), EBSCO, CINDOC (CSIC), CITEFACTOR, COPAC, DIALNET, DICE (CSIC), DOAJ, E-REVISTAS (CSIC), EBSCO PREMIER, ERIH+, GALE CENGAGE LEARNING, IN-RECS, IRESIE, LATINDEX, MIAR, OCLC WORLDCAT, RESH, REDIB, RILM CORE JOURNALS, SUDOC, ULRICHS, ESTA REVISTA ESTÁ PUBLICADA CON EL APOYO INSTITUCIONAL DE REDIRIS-CONSEJO SUPERIOR DE INVESTIGACIONES CIENTIFICAS Y ES DE ACCESO LIBRE. CREATIVE COMMONS LICENSE 4.O BY 
se contrastaron sus respuestas al finalizar el cuatrimestre cuando se repartió un cuestionario que, también, incluyó una pregunta sobre el tema.

Durante este período, también, contactamos con la responsable de los locales socioculturales del Ayuntamiento de Santiago de Compostela y se acordaron las fechas, locales y plazo de inscripción. Se llevó a cabo la difusión de la actividad mediante las redes sociales del Ayuntamiento, así como del blog del proyecto en el que se enmarcaron estas iniciativas (http://uniondecanlessociais.blogspot.com/). Además, se creó el material y se prepararon las diferentes sesiones, realizando reuniones con el alumnado de Grado que participaría posteriormente en el desarrollo de los talleres. Durante este período, desde la segunda edición, se solicitó al estudiantado universitario dos fotografías tamaño carnet, fotocopia de su DNI, el certificado de delitos sexuales y una ficha cubierta con sus datos para entregar al Servicio de Participación e Integración Universitaria de la propia institución, que fue el encargado de gestionar el seguro correspondiente.

\subsubsection{Segunda fase: implementación de los talleres}

En esta segunda fase, se desarrollaron los talleres que fueron diseñados en la fase anterior. Cada día en el que había programada una sesión, el alumnado de grado participante se reunía con la coordinadora de los talleres (alumna de $4^{\circ}$ curso con una Beca de Colaboración del Ministerio de Educación, Cultura y Deporte) o una de las profesoras en la Facultad de Ciencias de la Educación para recoger el material necesario y desplazarse hasta el lugar de la actividad.

Una vez allí, se procedía a organizar el espacio hasta que llegasen los participantes, momento en el que daba comienzo la actividad. Al final de la misma se realizaba una evaluación de la sesión a través de un cuestionario escrito con preguntas abiertas sobre su opinión acerca de las actividades realizadas, cambios que proponían y valoración global de la sesión en una escala del 1 al 10 y una rueda de opiniones, se despedía al alumnado participante y se recogía todo el material empleado, dejándolo nuevamente en la facultad. Posteriormente, y antes de la siguiente sesión, se subía al blog una pequeña noticia en la que se relataban las actividades realizadas, acompañadas de varias imágenes. Asimismo, se enviaban estas fotografías a la responsable de los Locales Socioculturales del Rural, encargada de difundirlas en las redes sociales. Además, al principio y al final de los talleres, se publicaron noticias sobre el proyecto, tanto en el periódico de la Universidad de Santiago de Compostela como en La Voz de Galicia.

\subsubsection{Tercera fase: evaluación}

En esta tercera fase, se realizó una evaluación de todas las acciones llevadas a cabo, con la finalidad de detectar fortalezas y debilidades que nos permitieran realizar los cambios oportunos en las siguientes ediciones de esta iniciativa.

La evaluación del alumnado participante se llevó a cabo durante todo el proceso, cubriendo un cuestionario al finalizar cada una de las sesiones en el que indicaban las actividades 
que más les habían gustado, las que menos, sugerencias, etc. Para complementar esta información recopilada, al final del taller los participantes realizaron una evaluación global del conjunto de las sesiones en las que, además de las actividades diseñadas, evaluaban al alumnado de Grado que los había estado acompañando, utilizando para esto un cuestionario en el que marcaban el grado de satisfacción del 1 al 10 con una serie de ítems o contestaban de forma abierta a distintas preguntas sobre las actividades desarrolladas en los talleres. Del mismo modo, tanto el alumnado de Grado como las responsables que participaron en esta experiencia de Aprendizaje-Servicio, también, cubrieron un cuestionario al finalizar la actividad en el que se recogía el grado de satisfacción, los aprendizajes extraídos vinculados a su futura profesión o los cambios que introducirían en el desarrollo de los talleres.

\section{Resultados}

\subsection{Percepción de los estudiantes universitarios}

La mayoría de los estudiantes universitarios evaluó positivamente la existencia del proyecto y la posibilidad de participar en él, y agradeció al profesorado la organización de la iniciativa. Así lo expresó, por ejemplo, una alumna que cursaba además estudios de piano en el conservatorio: "darte las gracias una vez más por aportar tanta confianza en mí, y permitirme participar en todas las iniciativas" (S.P.S, estudiante curso 2017/18). Asimismo, indicaron que estas actividades eran importantes como factor catalizador en la confirmación de sus vocaciones: "Este tipo de actividades son muy interesantes y gratificantes, por lo que deberían potenciarse, ya que nos ayudan a confirmar que esto es lo que realmente nos gusta" (S.P.S, C.O.M. y P.R.M., estudiantes curso 2017/18).

La organización de los talleres permitió al estudiantado familiarizarse con las exigencias que suponía planificar una sesión: qué actividades podían realizar para iniciar una clase y conocer a los participantes, qué actividades desarrollarían en la parte central y qué actividades podían usar para la vuelta a la calma o cierre (contribuyendo a desarrollar la competencia E54 del Plan de Estudios). En este sentido, la diferencia en las edades del alumnado y el número reducido de participantes supuso una dificultad añadida: "Al comienzo fue un poco complicado escoger las actividades y juegos para los niños y niñas por las edades diversas y el número de alumnos. A pesar de esto, creemos que la sesión salió bastante bien (...) participaron todos, integrándose muy bien" (A.M.R., M.A.N. y L.T.G., estudiantes curso 2016/17). También, se dieron cuenta de la importancia de organizar el espacio: por ejemplo, en la tercera edición, para evitar las distracciones de los niños que querían jugar al fútbol, retiraron unas pelotas que estaban colocadas en una estantería a la vista de todos. "Al llegar, lo primero que hicimos, después de transportar todo el material pertinente, fue guardar las pelotas que ocupaban una estantería del espacio asignado, en diferentes bolsas, debido a que estas causan numerosas distracciones en los niños" (S.P.S., C.O.M. y P.R.M., estudiantes curso 2017/18). Por otra parte, también, tomaron conciencia de la importancia de tener en cuenta los gustos y preferencias de los niños y niñas, en palabras de una estudiante de la primera edición de los talleres:

CAROL GILLANDERS, ANTIA CORES TORRES Y LAURA TOJEIRO PÉREZ. THE CONTENT OF THIS ARTICLE IS THE SOLE RESPONSIBILITY OF THE AUTHORS. THE REVISTA ELECTRÓNICA DE LEEME AND UNIVERSITAT DE VALĖNCIA ARE NOT LIABLE FOR ANY LEGAL ACTIONS THAT MAY ARISE INVOLVING THE ARTICLE'S CONTENT. REVISTA ELECTRÓNICA DE LEEME -LISTA ELECTRÓNICA EUROPEA DE MÚSICA EN LA EDUCACIÓN-HTTP://OJS.UV.ES/INDEX.PHP/LEEME/INDEX ISSN: 1575-9563. EDITORES: UNIVERSIDAD DE VALENCIA Y JESÚS TEJADA GIMÉNEZ. VISIBILIDAD DE ESTA REVISTA: SCOPUS, EMERGING CITATION INDEX (CLARIVATE), EBSCO, CINDOC (CSIC), CITEFACTOR, COPAC, DIALNET, DICE (CSIC), DOAJ, E-REVIISTAS ESTÁ PUBLICADA CON EL APOYO INSTITUCIONAL DE REDIRIS-CONSEJO SUPERIOR DE INVESTIGACIONES CIENTIFICAS Y ES DE ACCESO LIBRE. CREATIVE COMMONS LICENSE 4 . O BY 
"Es fundamental aprovechar sus gustos e intereses para encaminar la clase hacia el éxito. Este hecho fue palpable en una actividad en la que tenían que cantar (algo que a ellos y ellas no les gustaba), lo que implicó que tuviéramos que guiar rápidamente la actividad a los ritmos con percusión (algo que sí les atraía)" (E.F.G., estudiante curso 2015/16).

Otro ejemplo fue el vivenciado en el último taller, al evitar las distracciones de los participantes en las transiciones entre actividades y las discusiones entre ellos a través del diseño del taller como un juego ("Musiolimpíadas") en el que había que superar diferentes pruebas en busca de un tesoro. Incluir la gamificación en la sesión había sido propuesto por un grupo anterior de la materia de 'Motricidad Infantil' y había dado muy buenos resultados. En este caso, darles unas pegatinas en forma de gemas preciosas para colocar en sus ropas si superaban las pruebas hizo que el ambiente cambiara radicalmente. Así lo explican las estudiantes de Grado participantes: "El hecho de haber repartido 'puntos' en forma de pegatinas a lo largo de prácticamente todas las actividades promoviendo poco a poco su trabajo, con el resultado de un ‘tesoro’ final, fomentó su participación” (M.C.P. y S.F.R., estudiantes curso 2017/18).

El alumnado descubrió instrumentos para el aula de música que no conocía (flauta de émbolo o tambor oceánico) y las posibilidades que ofrecía el incluir espacios para la improvisación y la exploración de instrumentos, a través de la sonorización de diferentes situaciones o fotografías mediante la utilización de objetos diversos (piñas, palos de lluvia, conchas, etc.), consiguiendo desarrollar la competencia E57 del Plan de Estudios especificado en el apartado anterior. Así lo expresaba una de las alumnas:

\footnotetext{
"Descubrí las posibilidades musicales que nos pueden aportar objetos de la vida cotidiana (una bolsa, el palo de lluvia, los cotidiáfonos que semejan los truenos...). Me di cuenta de las ganas que tienen los niños/as de evadirse y experimentar con los instrumentos de formas muy diversas, por lo que resulta necesario dejarles tiempo libre para su disfrute y para el fomento de la creatividad" (E.F.G., estudiante curso 2015/16).
}

\subsection{Percepción de las docentes de la asignatura}

El alumnado que cursa la materia 'Música en la Educación Primaria' del Grado de Maestro/a en Educación Primaria presenta, por lo general, un nivel básico de dominio de las habilidades musicales. Según las respuestas obtenidas a nuestra pregunta sobre sus estudios musicales (al comenzar y finalizar la materia), durante el curso académico 2015/16 sólo un 5,1\% de nuestros estudiantes tenía estudios musicales de conservatorio o escuela de música, aumentando este porcentaje hasta un $8,3 \%$ si se incluían los estudiantes con estudios de música y danza tradicional gallega. En el curso 2016/17, se observó un incremento: en el primer caso hasta un $7 \%$ y, en el segundo, hasta un $17 \%$. Por último, de los participantes de la tercera edición, el $15 \%$ tocaba (o tocó de niño/a) algún instrumento musical y un $7 \%$ bailaba o tocaba música tradicional gallega.

Durante nuestras observaciones, pudimos constatar que todos los participantes universitarios demostraron una gran implicación en el proyecto, iniciativa que permitió a los estudiantes del Grado de Maestro/a de Educación Primaria desarrollar algunas de las

CAROL GILLANDERS, ANTIA CORES TORRES Y LAURA TOJEIRO PÉREZ. THE CONTENT OF THIS ARTICLE IS THE SOLE RESPONSIBILITY OF THE AUTHORS. THE REVISTA ELECTRÓNICA DE LEEME AND UNIVERSITAT DE VALĖNCIA ARE NOT LIABLE FOR ANY LEGAL ACTIONS THAT MAY ARISE INVOLVING THE ARTICLE'S CONTENT. REVISTA ELECTRÓNICA DE LEEME - LISTA ELECTRÓNICA EUROPEA DE MÚSICA EN LA EDUCACIÓN-HTTP://OJS.UV.ES/INDEX.PHP/LEEME/INDEX ISSN: 1575-9563. EDITORES: UNIVERSIDAD DE VALENCIA Y JESÚS TEJADA GIMÉNEZ. VISIBILIDAD DE ESTA REVISTA: SCOPUS, EMERGING CITATION INDEX (CLARIVATE), EBSCO, CINDOC (CSIC), CITEFACTOR, COPAC, DIALNET, DICE (CSIC), DOAJ, E-REVISTAS (CSIC), EBSCO PREMIER, ERIH+, GALE CENGAGE LEARNING, IN-RECS, IRESIE, LATINDEX, MIAR, OCLC WORLDCAT, RESH, REDIB, RILM CORE JOURNALS, SUDOC, ULRICHS, ESTA REVISTA ESTÁ PUBLICADA CON EL APOYO INSTITUCIONAL DE REDIRIS-CONSEJO SUPERIOR DE INVESTIGACIONES CIENTIFICAS Y ES DE ACCESO LIBRE. CREATIVE COMMONS LICENSE 4.O BY 
competencias y habilidades necesarias para la docencia, en este caso, planificar sesiones, implementar las actividades diseñadas, controlar y gestionar un grupo, tomar decisiones para adecuar su trabajo a las demandas del alumnado en determinadas situaciones, y reflexionar sobre la actividad en sí. Es importante destacar que el horario de los talleres no coincidía con su horario en la facultad; sin embargo, este hecho no supuso un impedimento para su participación.

Podemos afirmar que los estudiantes sin conocimientos musicales adquiridos fuera del espacio escolar se desenvolvían muy bien en los niveles 1 a 3 de desarrollo musical propuesto por Swanwick-Tillman (cit. en Runfola y Swanwick, 2002), planteando juegos y sonorizaciones que permitían explorar instrumentos escolares, crear atmósferas, seguir el pulso de diferentes músicas a través de diferentes juegos (aunque, a veces, se confundían los conceptos pulso y acento) y bailar diferentes danzas con una coreografía ya establecida. Pero, cuando propusimos realizar círculos de percusión con diferentes patrones (nivel 4 de la espiral de Swanwick-Tillman), tuvimos que recurrir a los estudiantes de conservatorio o escuela de música; también, fueron estos estudiantes los encargados de realizar las presentaciones de los instrumentos que no son escolares (clarinete, acordeón, saxofón, etc.).

Para el profesorado universitario que participó por primera vez en la tercera edición, el proyecto le permitió colaborar con sus colegas e intercambiar experiencias sobre la formación relacionada con el movimiento y la danza a la vez que le posibilitó participar en un proyecto de innovación docente, requisito imprescindible hoy en día para optar a la acreditación a los cuerpos de titular de universidad.

En relación a los beneficios de estos programas para los usuarios de los locales socioculturales del rural, podemos afirmar que los participantes de los talleres los valoraron muy positivamente, tanto en cada una de las evaluaciones semanales (cubiertas en la primera y tercera edición) como en la evaluación final. Según sus respuestas, las actividades que más éxito tuvieron fueron las más lúdicas (los juegos realizados siempre al inicio de la sesión), aquellas en las que debían sonorizar alguna situación, la presentación de instrumentos (violín, flauta travesera, etc.) y la experimentación con diferentes objetos.

\section{Conclusiones}

El análisis realizado sugiere que la participación en este tipo de iniciativas tiene un impacto positivo tanto en los estudiantes como en el profesorado. Sin embargo, es importante tener en cuenta las limitaciones de este estudio que, al no permitir generalizaciones, hace necesario realizar investigaciones en otros contextos de educación superior.

Proponemos que las experiencias desarrolladas promuevan los procesos de musicalización, es decir, "volver al individuo sensible y receptivo al fenómeno sonoro promoviendo en él al mismo tiempo, respuestas de índole musical” (Hemsy de Gainza, 2002, p. 133). En estos momentos, en donde ciertos sectores plantean la necesidad de abrir los concursos de profesorado universitario a las titulaciones de Grado de Maestro/a, abiertos hasta el momento a las titulaciones de Profesor/a Superior de Música en sus distintas especialidades, nuestra 
investigación sugiere que esta titulación no provee a los estudiantes sin una formación musical específica de las herramientas ni del conocimiento del contenido necesario para impartir clases de música más allá de una iniciación musical superficial. En cualquier caso, estos estudiantes sí pueden proponer actividades y juegos que estimulen habilidades musicales iniciales. Por este motivo, en la tercera edición procuramos que en cada una de las sesiones estuviera presente, al menos, una alumna con estudios musicales reglados.

Destacamos que la participación en este programa ayudó al alumnado sin una formación musical complementaria a la recibida en la escuela a ver las posibilidades que ofrecía la música para el desarrollo integral del niño (contribuyendo así a trabajar la competencia E54 del Plan de Estudios detallado en el apartado 2). Como sucedió en el estudio de Hennessy (2000), los estudiantes demostraron seguridad al realizar actividades que replicaban lo trabajado en las clases universitarias, en este caso, relacionar la música con la concienciación ambiental. Gracias al tratamiento musical de diversos contenidos de educación ambiental (reciclaje, sonidos de la naturaleza, cuidado del entorno, concienciación sobre el ruido, paisaje sonoro, etc.) el alumnado desarrolla un aprendizaje experiencial. Esta aproximación resulta especialmente importante en el medio rural, en el cual el contacto con la naturaleza es mucho más directo y significativo. De este modo, además de conectar con la realidad cotidiana del alumnado, esta experiencia permitió enseñar buenas prácticas para el cuidado del entorno, de modo que el alumnado se convierta en un ejemplo para el resto de la comunidad rural. Carrillo et al. (2017) argumentan que las investigaciones sobre la dimensión social de la educación musical pueden "dar pistas sobre la orientación que debería seguir la educación musical cursada en la enseñanza obligatoria para tener un impacto real sobre la sociedad" (p. 72). Como señalan Aróstegui y Cisneros-Cohernour (2010) "el debate está en el balance que hay que encontrar entre la formación como docente y como músico, es decir, entre los contenidos y las estrategias de enseñanza de los mismos" (p. 6).

Para el profesorado, esta iniciativa permitió integrar en su materia prácticas innovadoras que ayudan al cambio metodológico en la enseñanza superior. Las reflexiones llevadas a cabo nos permiten ver que, inconscientemente, mediante la implementación de los proyectos de ApS, estamos posiblemente intentando luchar contra la percepción general de la importancia de las artes en la educación y demostrar la relevancia curricular de la música. Se trata de una de las tres razones que mencionan Kezar y Rhoads (2001) como posibles causas del creciente interés en esta metodología. Comprobamos que, efectivamente, sí aporta un aire fresco al aula y otorga un nuevo impulso al profesorado (Bringle y Hatcher, 1996), ayudando al alumnado a entender el sentido de la materia. También, las grabaciones realizadas de las diferentes actividades son de una gran utilidad como material docente, puesto que a través de las mismas se ejemplifican propuestas de enseñanza de la música para estudiantes en edad escolar.

No queremos que la inclusión del ApS en nuestra materia sea efímera, tal como advierte Farmer (1990), sino que pretendemos seguir trabajando en este programa en los próximos años. La colaboración con la responsable de los locales socioculturales del rural de Santiago de Compostela ha sido clave para poder llevar a cabo las tres ediciones en las que se ha basado este estudio, y ya estamos organizando la cuarta. En nuestro caso, también contamos con apoyo institucional, dado que desde la propia universidad se ofrecen cursos de formación sobre el

CAROL GILLANDERS, ANTIA CORES TORRES Y LAURA TOJEIRO PÉREZ. THE CONTENT OF THIS ARTICLE IS THE SOLE RESPONSIBILITY OF THE AUTHORS. THE REVISTA ELECTRÓNICA DE LEEME AND UNIVERSITAT DE VALĖNCIA ARE NOT LIABLE FOR ANY LEGAL ACTIONS THAT MAY ARISE INVOLVING THE ARTICLE'S CONTENT. REVISTA ELECTRÓNICA DE LEEME -LISTA ELECTRÓNICA EUROPEA DE MÚSICA EN LA EDUCACIÓN-HTTP://OJS.UV.ES/INDEX.PHP/LEEME/INDEX ISSN: 1575-9563. EDITORES: UNIVERSIDAD DE VALENCIA Y JESÚS TEJADA GIMENEZ. VISIBILIDAD DE ESTA REVISTA: SCOPUS, EMERGING CITATION INDEX (CLARIVATE), EBSCO, CINDOC (CSIC), CITEFACTOR, COPAC, DIALNET, DICE (CSIC), DOAJ, E-REVISTAS
(CSIC), EBSCO PREMIER, ERIH+, GALE CENGAGE LEARNING, IN-RECS, IRESIE, LATINDEX, MIAR, OCLC WORLDCAT, RESH, REDIB, RILM CORE JOURNALS, SUDOC, ULRICHS, ESTA REVISTA ESTÁ PUBLICADA CON EL APOYO INSTITUCIONAL DE REDIRIS-CONSEJO SUPERIOR DE INVESTIGACIONES CIENTIFICAS Y ES DE ACCESO LIBRE. CREATIVE COMMONS LICENSE 4.O BY 
Aprendizaje-Servicio y se facilita la tramitación del seguro del estudiantado que debe desplazarse fuera de las instalaciones de la facultad. Asimismo, contamos con la colaboración del grupo de investigación ESCULCA, que realiza un seguimiento de nuestros proyectos.

Este tipo de iniciativas pueden ser fácilmente transferibles a otras instituciones de enseñanza superior que imparten una materia similar. Por ejemplo, gracias a la buena acogida de este proyecto, hemos organizado un taller de música en la Fundación Down en otra materia que impartimos en la mención de Educación Musical, y ampliamos la oferta no sólo a los locales socioculturales del rural sino también a la guardería de la propia universidad para el alumnado que cursará la mención en lengua extranjera, con el fin de realizar un taller de música en inglés.

\section{Referencias}

Aróstegui, J. L. y Cisneros-Cohernour, E. (2010). Reflexiones en torno a la formación del profesorado de música a partir del análisis documental de los planes de estudio en Europa y América Latina. Profesorado. Revista de Currículum y Formación de Profesorado, 14 (2), 179189. Recuperado de https://recyt.fecyt.es/

Bamford, A. (2009). The Wow Factor. Global research compendium on the impact of the arts in education. Münster: Waxmann Verlag GmbH.

Bautista Cupul, P. (2017). El futuro maestro generalista ¿preparado para utilizar la música en su enseñanza? Revista Educación y Ciencia, 6 (47), 32-44. Recuperado de http://educacionyciencia.org/

Bresler, L. (2002). Research: A Foundation for Arts Education Advocacy. En R. Colwell y C. Richardson (Eds.), The New Handbook of Research on Music Teaching and Learning (pp.10661083). Nueva York: Oxford University Press.

Bringle, R. G. y Hatcher, J. A. (1996). Implementing Service Learning in Higher Education. The Journal of Higher Education, 67 (2), 221-239. doi: 10.1080/00221546.1996.11780257

Bringle, R. G. y Hatcher, J. A. (2000). Institutionalization of Service Learning in Higher Education. The Journal of Higher Education, 71 (3), 273-290. doi: 10.2307/2649291

Burke, K. (2016). Rising to the challenge: Supporting educators without arts experience in the delivery of authentic arts learning. AARE Conference 2016. Melbourne, Victoria. Recuperado de https://www.aare.edu.au/

Bustamante, E. (Coord.) (2016). Informe sobre el estado de la cultura en España 2016. Madrid: Fundación Alternativas.

CAROL GILLANDERS, ANTIA CORES TORRES Y LAURA TOJEIRO PÉREZ. THE CONTENT OF THIS ARTICLE IS THE SOLE RESPONSIBILITY OF THE AUTHORS. THE REVISTA ELECTRÓNICA DE LEEME AND UNIVERSITAT DE VALËNCIA ARE NOT LIABLE FOR ANY LEGAL ACTIONS THAT MAY ARISE INVOLVING THE ARTICLE'S CONTENT. REVISTA ELECTRÓNICA DE LEEME - ISTA ELECTRÓNICA EUROPEA DE MÚSICA EN LA EDUCACIÓN-HTTP://OJS.UV.ES/INDEX.PHP/LEEME/INDEX ISSN: 1575-9563. EDITORES: UNIVERSIDAD DE VALENCIA Y JESÚS TEJADA GIMÉNEZ. VISIBILIDAD DE ESTA REVISTA: SCOPUS, EMERGING CITATION INDEX (CLARIVATE), EBSCO, CINDOC (CSIC), CITEFACTOR, COPAC, DIALNET, DICE (CSIC), DOAJ, E-REVISTAS (CSIC), EBSCO PREMIER, ERIH+, GALE CENGAGE LEARNING, IN-RECS, IRESIE, LATINDEX, MIAR, OCLC WORLDCAT, RESH, REDIB, RILM CORE JOURNALS, SUDOC, ULRICHS, ESTA REVISTA ESTÁ PUBLICADA CON EL APOYO INSTITUCIONAL DE REDIRIS-CONSEJO SUPERIOR DE INVESTIGACIONES CIENTIFICAS Y ES DE ACCESO LIBRE. CREATIVE COMMONS LICENSE 4.O BY 
Carrillo, C., Viladot, L. y Pérez-Moreno, J. (2017). Impacto de la educación musical: una revisión de la literatura científica. Revista Electrónica Complutense de Investigación en Educación Musical, 14, 61-74. doi: 10.5209/RECIEM.54828

Creswell, J. W. (1998). Qualitative inquiry and research design. Choosing among five traditions. London: Sage Publications Inc.

Deasy, R. J. (Ed.) (2002). Critical Links; Learning in the Arts and Student Academic and Social Development. Washington DC: Arts Education Partnership.

Dogani, K. (2008). Using reflection as a tool for training generalist teachers to teach music de Konstantina Dogani. Music Education Research, 10 (1), 125-139. doi: 10.1080/14613800701871595

Ewing, R. (2010). The arts and Australian education: Realistic potential. Victoria: Australian Council for Educational Research.

Farmer, D. W. (1990). Strategies for Change. En D. W. Steeples (Ed.). Managing Change in Higher Education (pp. 7-17). San Francisco: Jossey-Bass.

Fiske, E. (Ed.) (1999). Champions of change: The impact of the arts on learning. Washington DC: Arts Education Partnership.

Jefatura del Estado. Ley Orgánica 8/2013, de 9 de diciembre, para la mejora de la calidad educativa. BOE (10/12/2013), no 295, referencia 12886, pp. 97858-97921.

Giordanelli, R. M. (2011). La música en la educación, herramienta fundamental para la formación integral. El Astrolabio, 59, 58-67. Recuperado de https://es.scribd.com/

Hemsy de Gainza, V. (2002). Música: Amor y conflicto. Diez estudios de psicopedagogía musical. Buenos Aires: Ed. Lumen.

Hennessy, S. (2000). Overcoming the red-feeling: The development of confidence to teach music in primary school amongst student teachers. British Journal of Music Education, 17 (2), 183-196. Recuperado de https://www.cambridge.org/

Kezar, A. y Rhoads, R.A. (2001). The Dynamic Tensions of Service Learning in Higher Education: A Philosophical Perspective. The Journal of Higher Education, 72 (2), 148-171. doi: $10.2307 / 2649320$

López García, N., Madrid Vivar, D. y De Moya Martínez, M. V. (2017). La formación musical en los planes de estudio para maestros de Primaria en la Universidad de Castilla-La Mancha. Estudios Pedagógicos, 43 (1), 423-438. doi: 10.4067/S0718-07052017000100024

López García, N., y De Moya Martínez, M.V. (2017). Documentos clave de la Unión Europea sobre educación musical en las enseñanzas obligatorias. Revista Electrónica Complutense de Investigación en Educación Musical, 14, 171-186. doi: 10.5209/RECIEM.52409

CAROL GILLANDERS, ANTIA CORES TORRES Y LAURA TOJEIRO PÉREZ. THE CONTENT OF THIS ARTICLE IS THE SOLE RESPONSIBILITY OF THE AUTHORS. THE REVISTA ELECTRÓNICA DE LEEME AND UNIVERSITAT DE VALĖNCIA ARE NOT LIABLE FOR ANY LEGAL ACTIONS THAT MAY ARISE INVOLVING THE ARTICLE'S CONTENT. REVISTA ELECTRÓNICA DE LEEME -LISTA ELECTRÓNICA EUROPEA DE MÚSICA EN LA EDUCACIÓN-HTTP://OJS.UV.ES/INDEX.PHP/LEEME/INDEX ISSN: 1575-9563. EDITORES: UNIVERSIDAD DE VALENCIA Y JESÚS TEJADA GIMÉNEZ. VISIBILIDAD DE ESTA REVISTA: SCOPUS, EMERGING CITATION INDEX (CLARIVATE), EBSCO, CINDOC (CSIC), CITEFACTOR, COPAC, DIALNET, DICE (CSIC), DOAJ, E-REVISTAS (CSIC), EBSCO PREMIER, ERIH+, GALE CENGAGE LEARNING, IN-RECS, IRESIE, LATINDEX, MIAR, OCLC WORLDCAT, RESH, REDIB, RILM CORE JOURNALS, SUDOC, ULRICHS, ESTA REVISTA
ESTÁ PUBLICADA CON EL APOYO INSTITUCIONAL DE REDIRIS-CONSEJO SUPERIOR DE INVESTIGACIONES CIENTIFICAS Y ES DE ACCESO LIBRE. CREATIVE COMMONS LICENSE 4.0 BY 
Lorenzo, Ma M., Mella, I., García, J. y Varela, C. (2017). Investigar para institucionalizar el aprendizaje servicio en la universidad española. RIDAS, Revista Iberoamericana de Aprendizaje Servicio, 3, 118-130. doi: 10.1344/RIDAS2017.3.9

Mark, M. L. (2002). Nonmusical Outcomes of Music Education: Historical Considerations. En R. Colwell y C. Richardson (Eds.). The New Handbook of Research on Music Teaching and Learning (pp. 1045-1052). New York: Oxford University Press.

Puig Rovira, J. M., Batlle, R., Bosch, C. y Palos, J. (2006). Aprenentatge servei. Educar per a la ciutadanía. Barcelona: Octaedro.

Puig Rovira, J. M., Gijón Casares, M., Martín García, X. y Rubio Serrano, L. (2011). Aprendizajeservicio y Educación para la Ciudadanía. Revista de Educación, Extra.1, 45-67. Recuperado de http://www.revistaeducacion.educacion.es/

Rauscher, F. H. (2009). The impact of music instrucion on other skills. En S. Hallam, I. Cross, y M. Thaut (Eds.), The Oxford Handbook of Music Psychology (pp. 244-252). Nueva York: Oxford University Press. doi: 10.1093/oxfordhb/0780199298457.013.0023

Riaño Galán, M. E., Mier Pérez, P. y Pozo Miranda, M. (2017). Aprendizaje-servicio a través de la perfomance: análisis de una práctica artística para el desarrollo socio-emocional y creativo en la formación inicial del profesorado. Revista de Estudios y Experiencias en Educación, 16 (32), 151-164. doi: 10.21703/rexe.20173215116410

Rosa Napal, F. C. (2015). La formación musical del futuro profesorado de educación primaria (Tesis doctoral inédita). La Coruña: Universidad de La Coruña. Recuperado de http://ruc.udc.es/dspace/handle/2183/15952

Runfola, M. y Swanwick, K. (2002). Developmental characteristics of music learners. En R. Colwell y C. Richardson (Eds.). The New Handbook of Research on Music Teaching and Learning (pp. 373-397). New York: Oxford University Press.

Sadio-Ramos, F. J. y Ortiz-Molina, M. A. (2018). ContiTuna: Intervención sociocultural por medio de la práctica musical popular. Modulema. Revista Científica sobre Diversidad Cultural, 2 (1), 7-29. Recuperado de http://revistaseug.ugr.es

Sáez, P. y Marín, D. (2016). Música y educación en valores: Aprendizaje servicio en la escuela primaria. Quaderns Digitals: Revista de Nuevas Tecnologías y Sociedad, 83, 34-43. Recuperado de https://dialnet.unirioja.es/

Santos, M. A., Sotelino Losada, A. y Lorenzo Moledo, M. (2015). Aprendizaje-servicio y misión cívica de la universidad. Una propuesta de desarrollo. Barcelona: Octaedro.

Sigmon, R. (1997). Linking Service with Learning in Liberal Arts Education. Washington: Council of Independent Colleges.

CAROL GILLANDERS, ANTÍA CORES TORRES Y LAURA TOJEIRO PÉREZ. THE CONTENT OF THIS ARTICLE IS THE SOLE RESPONSIBILITY OF THE AUTHORS. THE REVISTA ELECTRÓNICA DE LEEME AND UNIVERSITAT DE VALĖNCIA ARE NOT LIABLE FOR ANY LEGAL ACTIONS THAT MAY ARISE INVOLVING THE ARTICLE'S CONTENT. REVISTA ELECTRÓNICA DE LEEME -LISTA ELECTRÓNICA EUROPEA DE MÚSICA EN LA EDUCACIÓN-HTTP://OJS.UV.ES/INDEX.PHP/LEEME/INDEX ISSN: 1575-9563. EDITORES: UNIVERSIDAD DE VALENCIA Y JESÚS TEJADA GIMÉNEZ. VISIBILIDAD DE ESTA REVISTA: SCOPUS, EMERGING CITATION INDEX (CLARIVATE), EBSCO, CINDOC (CSIC), CITEFACTOR, COPAC, DIALNET, DICE (CSIC), DOAJ, E-REVISTAS (CSIC), EBSCO PREMIER, ERIH+, GALE CENGAGE LEARNING, IN-RECS, IRESIE, LATINDEX, MIAR, OCLC WORLDCAT, RESH, REDIB, RILM CORE JOURNALS, SUDOC, ULRICHS, ESTA REVISTA 
Siebenaler, D. (2005). Music and Service Learning: A Case Study. Journal of Music Teacher Education, 15 (1), 23-30. doi: 10.1177/10570837050150010105

Ugarte, C. y Naval, C. (2010). El service-learning, medio adecuado para promover la cooperación social. El caso concreto del programa Create your own charity. En C. Naval, S. Lara, C. Ugarte y C. Sádaba (Eds.). Educar para la comunicación y la cooperación social (pp. 263-281). Pamplona: Consejo Audiovisual de Navarra. Recuperado de http://dadun.unav.edu/

Universidade de Santiago de Compostela. (2011). Memoria para la verificación del título de Grado en Maestro/a de Educación Primaria. Recuperado de http://www.usc.es/ 